\title{
STAGNATION ZONES OF IDEAL FLOWS IN LONG AND NARROW BANDS
}

\author{
V. M. MIKLYUKOV, S.-S. CHOW, and V. P. SOLOVJOV
}

Received 31 October 2001

\begin{abstract}
We investigate stagnation zones of flows of ideal incompressible fluid in narrow and long bands. With the bandwidth being much less than its length, these flows are almost stationary over large subdomains, where their potential functions are almost constant. These subdomains are called $s$-zones. We estimate the size and the location of these $s$-zones.
\end{abstract}

2000 Mathematics Subject Classification: 28A75, 30C75, 35J25.

1. Introduction. In this work, we investigate the flows of ideal incompressible fluid in narrow and long bands on surfaces with almost Liouville line element. The investigation is motivated in part by the fact that, with the recent increasing interest in areas such as microelectromechanical systems (MEMS) and nanoscale physiological processes, there is a greater need to improve our understanding of fluid flows in the microscale and nanoscale regimes.

When the width of the band is much smaller than the length, the zones inside which the flows are almost stationary, and consequently their potential functions are almost constant, will be of sufficiently large size. Here, we study the size and location of these stagnation zones, which we will call $s$-zones of ideal flows. At first sight, it seems that the situation is of little interest. However, by remembering that minute change in potential function value occurs over a very long interval, it is clear that a better understanding of such $s$-zones may allow one to better organize calculations and possible minimize the amount of computation.

First, we define the following concept which will be key in this article.

DEFINITION 1.1. Let $f: \mathbf{D} \rightarrow \mathbb{R}$ be a continuous function. Fix a subdomain $U \subset \mathbf{D}$ and a constant $s>0$. The subdomain $U$ is said to be an $s$-zone of $f$ if the oscillation of $f$ on $U$ does not exceed the preassigned constant $s$.

For any given continuous function $f: \mathbf{D} \rightarrow \mathbb{R}$, and for arbitrary $s>0$, it is easy to see that every point in $\mathbf{D}$ will have a neighborhood which is an $s$-zone. Here, we are mainly interested in $s$-zones that are sufficiently large; intuitively, we are interested in $s$-zones whose sizes or measures are of the same order as the size of $\mathbf{D}$. The presence of such $s$-zones can be an obstacle in numerical computation when, for example, the order $s$ is comparable with machine epsilon in the floating point system. A priori knowledge of the size and location of $s$-zones in $\mathbf{D}$ allows one to better organize and focus computational effort on regions where the solution is rapidly varying. It may also lead to significant 
reduction in the computational requirements of numerical solution of the boundary value problem.

In this paper, we consider ideal incompressible flows in long and narrow bands over surfaces with an almost Liouville line element (see, e.g., [5, Section 5.7.5]). Similar problems were studied over surfaces with line elements in $[7,8]$. In these works, the $s$-zones were shown to be related to some problems in the construction of conformal mappings from long rectangles to a unit disk $[4,6,11]$ and to some problems arising in economics [2, Chapter I]. In this paper, we provide estimates for the size of $s$-zones. The main results are given in Section 3 and some examples are provided in the last section.

2. Boundary value problems. To begin our study, we first introduce some notations to define long and narrow bands and almost Liouville line elements and discuss the boundary value problem associated with ideal incompressible flows in bands over surfaces with almost Liouville line elements.

Let $\theta: \mathbb{R}^{2} \rightarrow \mathbb{R}$ be a given Lipschitz function. Let $\mathbf{D}$ be a domain in $\mathbb{R}^{2}$ consisting of points $(x, y)$ such that $0<\theta(x, y)<1$, and with the property that for any bounded subdomain $D^{\prime}$ whose closure is contained in $\mathbf{D}$,

$$
\text { essinf } \operatorname{Din}_{D^{\prime}}|\nabla \theta(x, y)|>0 \text {. }
$$

For $0 \leq \tau \leq 1$, let

$$
\Gamma_{\tau}=\{(x, y) \in \mathbf{D}: \theta(x, y)=\tau\}
$$

We will assume the boundary curves $\Gamma_{0}$ and $\Gamma_{1}$ connected and of unlimited extent.

Let $\gamma(\tau)$ denote the connected component of the set

$$
\{(x, y) \in \mathbf{D}: y=\tau\}, \quad-\infty<\tau<+\infty,
$$

which separates the subregion of $D$ with $y \approx-\infty$ from the subregion of $D$ with $y \approx \infty$. This would ensure the band described below to be well defined. For $t>0$, a band of width $t$ is defined as the subdomain $D(t)$ of $\mathbf{D}$ bounded by $\Gamma_{0}, \Gamma_{1}, \gamma(-t)$ and $\gamma(t)$. The band is long and narrow when the width is small compared to its length, that is, $t \ll 1$.

Let $\Gamma_{s}(t)=\Gamma_{s} \cap \overline{D(t)}, 0 \leq s \leq 1$. In what follows, let $\beta>0$ be a given parameter.

The model boundary value problems that we are interested in consist of an equation describing harmonic functions on surfaces with almost Liouville line elements and either Dirichlet, Neumann, or mixed boundary conditions. Let $D \subset \mathbb{R}^{2}$ be a domain at two-dimensional Euclidean plane with a coordinate system $(x, y)$, and let $A, B$, and $Q$ be some functions on $D$. Suppose that $A, B, Q>0$ everywhere on $D$. A line element of the form

$$
d s^{2}=Q^{2}(x, y)\left(A^{2}(x) d x^{2}+B^{2}(y) d y^{2}\right), \quad(x, y) \in D,
$$

is called an almost Liouville line element. An area element on the surface $\mathscr{D}=\left(D, d s^{2}\right)$ is given by

$$
d \sigma=Q^{2}(x, y) A(x) B(y) d x d y .
$$


In the case where $Q(x, y)$ has the special form $Q^{2}(x, y)=\alpha(x)-\beta(y)$, with $\alpha, \beta$ being arbitrary functions such that $\alpha>\beta$, we recover the standard definition of a Liouville line element (see, e.g., [5, Section 5.7.5]). An example of surfaces defined by an almost Liouville line element is given by the class of second-order confocal surfaces [5, Section 3.7.3]. Further examples of almost Liouville line elements will be given in Section 7 .

On surfaces with almost Liouville line elements, we consider the following LaplaceBeltrami equation in a band $D(\beta)$ :

$$
B(y) \frac{\partial}{\partial x}\left(A(x)^{-1} \frac{\partial f}{\partial x}\right)+A(x) \frac{\partial}{\partial y}\left(B(y)^{-1} \frac{\partial f}{\partial y}\right)=0
$$

and one of the following boundary conditions:

$$
\left.f\right|_{\Gamma_{i}(\beta)}=0 \quad(i=0,1)
$$

or

$$
A^{-1} B f_{x}^{\prime} \cos v+\left.A B^{-1} f_{y}^{\prime} \sin v\right|_{\Gamma_{i}(\beta)}=0 \quad(i=0,1)
$$

or

$$
\left.f\right|_{\Gamma_{0}(\beta)}=0, \quad A^{-1} B f_{x}^{\prime} \cos v+\left.A B^{-1} f_{y}^{\prime} \sin v\right|_{\Gamma_{1}(\beta)}=0
$$

Here, $v$ is an angle between a unit normal vector to $\partial D(\beta)$ and the $x$-axis, and $A, B$ are given functions related to the underlying line elements.

Note that on $\gamma(-\beta) \cup \gamma(\beta)$, the solution value of $f$ is not specified. We assume that additional boundary conditions will be given so that the boundary value problem is well posed.

3. Main results. We now state our main results. The proof will be given in Section 6 . The first theorem shows that, if the bandwidth is sufficiently small relative to its length, the solution $f$ to the boundary value problem discussed in the previous section will have sufficiently large $s$-zones. By "solution" here we mean generalized local Lipschitz solution, with the precise meaning to be stated in Section 4.

Let $f$ be a Lipschitz function on a band $D(\beta)$. For an arbitrary $t \in(1, \beta), \beta>1$, we set

$$
\begin{aligned}
\Omega & =\operatorname{osc}_{y(-\beta) \cup y^{(\beta)}} f(x, y), \\
e(t) & =\iint_{D(t)}\left(\frac{B}{A} \theta^{\prime 2}{ }_{x}+\frac{A}{B} \theta^{\prime 2}\right) d x d y, \\
\mu(t) & =\max \left\{\iint_{D^{*}} \frac{A}{B} d x d y, \iint_{D^{* *}} \frac{A}{B} d x d y\right\},
\end{aligned}
$$

where $D^{*}=D \cap\{-t<y<-t+1\}$ and $D^{* *}=D \cap\{t<y<t+1\}$,

$$
I(p, q)=\min \left\{\int_{-q}^{-p} \frac{B d y}{\int_{y(y)} A d x}, \int_{p}^{q} \frac{B d y}{\int_{\gamma(y)} A d x}\right\}, \quad 0<p<q \leq \beta .
$$


THEOREM 3.1. Let $f$ be a solution of (2.6) on $D(\beta)$ with one of conditions (2.7), (2.8), or (2.9). For any given $s>0$, if there exists $t$ such that $1<t<\beta$ and

$$
\frac{3}{2}(e(t)+\mu(t)) \frac{\Omega^{2}}{I(t, \beta)} \leq s^{2}
$$

then the region $D(t-1)$ is an $s$-zone of $f$.

Now, we denote

$$
\kappa(t)=\min \left\{\frac{B(-t)}{L(-t)}, \frac{B(t)}{L(t)}\right\}, \quad L(t)=\int_{\gamma(t)} A(x) d x
$$

We also have the following related result not involving $\Omega$ and $I(t, \beta)$.

THEOREM 3.2. Let $f$ be a solution of (2.6) on $D(\beta)$ satisfying either the boundary condition (2.7) or (2.9), and let $s>0$ be a given number. The region $D(t-2)$ is an $s$-zone of $f$ if there exists $t$ such that $2<t<\beta$ and

$$
\frac{3}{2 I(\beta-1, \beta)}(e(t-1)+\mu(t-1)) \exp \left\{-C \pi \int_{t-1}^{\beta-1} \kappa(\tau) d \tau\right\} \leq s^{2},
$$

where $C=2$ for the Dirichlet condition (2.7) and $C=1$ for the mixed boundary condition (2.9).

These theorems provide estimates for the size, or more specifically, the width, of an $s$-zone when the inequality (3.5) or (3.7) is satisfied.

4. Oscillation estimates. For an arbitrary subdomain $U \subset \mathbb{R}^{2}$, let Lip $U$ be the set of functions $f$ satisfying the Lipschitz condition

$$
\left|f\left(x^{\prime \prime}, y^{\prime \prime}\right)-f\left(x^{\prime}, y^{\prime}\right)\right| \leq C \sqrt{\left(x^{\prime \prime}-x^{\prime}\right)^{2}+\left(y^{\prime \prime}-y^{\prime}\right)^{2}}
$$

for all $\left(x^{\prime}, y^{\prime}\right),\left(x^{\prime \prime}, y^{\prime \prime}\right) \in U$ with a constant $C=C(f, U)$.

Technically, it is convenient to use the following definition of solutions of (2.6) with (2.7), (2.8), or (2.9). A function $f \in \operatorname{Lip} \overline{D(\beta)}$ is said to be the generalized solution of (2.6) with boundary condition (2.7), (2.8), or (2.9) if, for any arbitrary bounded subdomain $\Delta$, contained in $D(\beta)$, with a rectifiable boundary $\partial \Delta$ and any arbitrary function $\phi \in \operatorname{Lip} \bar{\Delta}$, we have

$$
\iint_{\Delta}\left(\phi_{x}^{\prime} \frac{B}{A} f_{x}^{\prime}+\phi_{y}^{\prime} \frac{A}{B} f_{y}^{\prime}\right) d x d y=\int_{\partial^{\prime} \Delta} \phi\left(-\frac{A}{B} f_{y}^{\prime} d x+\frac{B}{A} f_{x}^{\prime} d y\right)
$$

where $\partial^{\prime} \Delta=\partial \Delta \backslash\left(\Gamma_{0}(\beta) \cup \Gamma_{1}(\beta)\right)$. Note that in the above definition, at any point $x \in D$, where $\nabla f(x)$ does not exist, we set $\nabla f(x)=0$.

Let $A, B$ be functions of $C^{1}(\overline{D(\beta)})$ and let $f \in C^{2}(\overline{D(\beta)})$ be a solution of (2.6) on $D(\beta)$. By Green formula for any bounded domain $\Delta \subset D(\beta)$ with the rectifiable boundary and 
any function $\phi \in \operatorname{Lip} \bar{\Delta}$, we have

$$
\begin{aligned}
\iint_{\Delta} & \phi\left(\left(\frac{B}{A} f_{x}^{\prime}\right)_{x}^{\prime}+\left(\frac{A}{B} f_{y}^{\prime}\right)_{y}^{\prime}\right) d x d y+\iint_{\Delta}\left(\phi_{x}^{\prime} \frac{B}{A} f_{x}^{\prime}+\phi_{y}^{\prime} \frac{A}{B} f_{y}^{\prime}\right) d x d y \\
= & \int_{\partial^{\prime} \Delta}-\phi \frac{A}{B} f_{y}^{\prime} d x+\phi \frac{B}{A} f_{x}^{\prime} d y .
\end{aligned}
$$

From here, by (2.6), we obtain (4.2).

LEMMA 4.1. If $f$ is a generalized solution of (2.6) with boundary condition (2.7), (2.8), or (2.9) on $D(\beta)$, then for any $t, 0 \leq t \leq \beta$,

$$
\max _{D(t)} f(x, y)=\max _{\gamma(-t) \cup y^{(}(t)} f(x, y)
$$

Proof. Suppose there exists a point $\left(x_{0}, y_{0}\right) \in D(t)$ such that

$$
f\left(x_{0}, y_{0}\right)>\max _{\gamma(-t) \cup \gamma^{\prime}(t)} f(x, y)=M \text {. }
$$

Choose $\epsilon$ such that $f\left(x_{0}, y_{0}\right)>\epsilon>M$. Consider a connected component $\Delta$ of the set $\{(x, y) \in D(t): f(x, y)>\epsilon\}$. By (4.2) with $\phi=f(x, y)-\epsilon$, we can write

$$
\iint_{\Delta}\left(\frac{B}{A} f_{x}^{\prime 2}+\frac{A}{B} f_{y}^{\prime 2}\right) d x d y=\int_{\partial^{\prime} \Delta}(f-\epsilon)\left(-\frac{A}{B} f_{y}^{\prime} d x+\frac{B}{A} f_{x}^{\prime} d y\right)=0
$$

As $A, B>0$ everywhere on $\Delta$, we obtain

$$
\nabla f(x, y)=0 \quad \text { everywhere on } \Delta,
$$

and thus $f \equiv$ const on $\Delta$, which is a contradiction.

LEMMA 4.2. Let $t$ be a fixed number, $0<t \leq \beta$, and let $f$ be a generalized solution of (2.6) with boundary condition (2.7), (2.8), or (2.9) on $D(\beta)$. Then,

$$
\inf _{0 \leq s \leq 1} \operatorname{osc}_{\Gamma_{S}(t)}^{2} f \leq \iint_{D(t)} \mathscr{E}(\nabla \theta) d x d y \iint_{D(t)} \mathscr{E}(\nabla f) d x d y .
$$

Proof. Fix $s \in(0,1)$, and $t \in(0, \beta)$. Let $\left(x_{0}, y_{0}\right) \in \Gamma_{s}(t)$ be a fixed point. At this point, the vector

$$
\bar{\tau}=\left(-\frac{\theta_{y}^{\prime}}{|\nabla \theta|}, \frac{\theta_{x}^{\prime}}{|\nabla \theta|}\right)
$$

is a unit tangent to $\Gamma_{s}(t)$ vector, and

$$
\frac{\partial f}{\partial \bar{\tau}}=\langle\nabla f, \bar{\tau}\rangle
$$

is a derivative with respect to the direction $\bar{\tau}$.

Because the function $f$ is locally Lipschitz and almost all level curves $\Gamma_{s}(t), 0<$ $s<1$, of the Lipschitz function $\theta$ are rectifiable [3, Theorem 3.2.15], the function $f$ is 
absolutely continuous along $\Gamma_{s}(t)$ for almost all $s \in(0,1)$. From

$$
\begin{aligned}
\mathscr{E}(\nabla f) \mathscr{E}(\nabla \theta)-\left|\nabla \theta^{2}\right|\left|\frac{\partial f}{\partial \bar{\tau}}\right|^{2}= & \left(\frac{B}{A} f_{x}^{\prime 2}+\frac{A}{B} f_{y}^{\prime 2}\right)\left(\frac{B}{A} \theta_{x}^{\prime 2}+\frac{A}{B} \theta_{y}^{\prime 2}\right) \\
& -\left(\left(f_{x}^{\prime} \theta_{y}^{\prime}-f_{y}^{\prime} \theta_{x}^{\prime}\right)\right)^{2} \\
= & \left(\frac{B}{A} f_{x}^{\prime} \theta_{x}^{\prime}+\frac{A}{B} f_{y}^{\prime} \theta_{y}^{\prime}\right)^{2} \geq 0,
\end{aligned}
$$

we see that at $\left(x_{0}, y_{0}\right)$,

$$
\left|\frac{\partial f}{\partial \bar{\tau}}\right|^{2} \leq \frac{1}{|\nabla \theta|^{2}} \mathscr{E}(\nabla f) \mathscr{E}(\nabla \theta)
$$

Next, denoting $\sqrt{d x^{2}+d y^{2}}$ by $d \ell$, we note that

$$
\operatorname{osc}_{\Gamma_{S}(t)} f \leq \int_{\Gamma_{S}(t)}\left|\frac{\partial f}{\partial \bar{T}}\right| d \ell \leq \int_{\Gamma_{S}(t)} \mathscr{E}^{1 / 2}(\nabla f)^{\mathscr{E}^{1 / 2}}(\nabla \theta) \frac{d \ell}{|\nabla \theta|} .
$$

Using the Cauchy inequality, we obtain

$$
\operatorname{osc}_{\Gamma_{S}(t)}^{2} f \leq \int_{\Gamma_{S}(t)} \mathscr{E}(\nabla f) \frac{d \ell}{|\nabla \theta|} \int_{\Gamma_{S}(t)} \mathscr{E}(\nabla \theta) \frac{d \ell}{|\nabla \theta|}
$$

This inequality implies

$$
\int_{0}^{1} \frac{\operatorname{osc}_{\Gamma_{S}(t)}^{2} f}{\int_{\Gamma_{S}(t)} \mathscr{E}(\nabla \theta)(d \ell /|\nabla \theta|)} d s \leq \int_{0}^{1} \int_{\Gamma_{S}(t)} \mathscr{E}(\nabla f) \frac{d \ell}{|\nabla \theta|} d s .
$$

The condition (2.1) makes possible to use the coarea formula [3, Theorem 3.2.12]. We have

$$
\int_{0}^{1} \int_{\Gamma_{S}(t)} \mathscr{E}(\nabla f) \frac{d \ell}{|\nabla \theta|} d s=\iint_{D(t)} \mathscr{E}(\nabla f) d x d y
$$

Thus, we arrive at the following relation:

$$
\inf _{0 \leq s \leq 1} \operatorname{osc}_{\Gamma_{S}(t)}^{2} f \int_{0}^{1} \frac{d s}{\int_{\Gamma_{S}(t)} \mathscr{E}(\nabla \theta)(d \ell /|\nabla \theta|)} \leq \iint_{D(t)} \mathscr{E}(\nabla f) d x d y .
$$

Next, from the Cauchy inequality, it follows that

$$
1=\int_{0}^{1} d s \leq \int_{0}^{1} \frac{d s}{\int_{\Gamma_{S}(t)} \mathscr{E}(\nabla \theta)(d \ell /|\nabla \theta|)} \int_{0}^{1} \int_{\Gamma_{S}(t)} \mathscr{E}(\nabla \theta) \frac{d \ell}{|\nabla \theta|} d s
$$


Again, applying the coarea formula, we find

$$
1 \leq \int_{0}^{1} \frac{d s}{\int_{\Gamma_{s}(t)} \mathscr{E}(\nabla \theta)(d \ell /|\nabla \theta|)} \iint_{D(t)} \mathscr{E}(\nabla \theta) d x d y
$$

From here, with (4.17), we obtain (4.8).

LEMMA 4.3. Let $f$ be a generalized solution of (2.6) with boundary condition (2.8), (2.9), or (2.7) on $D(\beta)$ and let $\tau, t, 0<\tau<t<\beta$, be some arbitrary numbers. Then,

$$
\begin{aligned}
& \inf _{y \in(\tau, t)} \operatorname{osc}_{\gamma(y)}^{2} f+\inf _{y \in(-t,-\tau)} \operatorname{osc}_{\gamma(y)}^{2} f \\
& \quad \leq \frac{1}{(t-\tau)^{2}} \max \left\{\iint_{D^{\prime}} \frac{A}{B} d x d y, \iint_{D^{\prime \prime}} \frac{A}{B} d x d y\right\} \iint_{D(t) \backslash D(\tau)} \mathscr{E}(\nabla f) d x d y,
\end{aligned}
$$

where $D^{\prime}=D(\beta) \cap\{-t<y<-\tau\}$ and $D^{\prime \prime}=D(\beta) \cap\{\tau<y<t\}$.

Proof. We will show that Lemma 4.3 may be obtained as a corollary of Lemma 4.2. Choosing $\theta_{0}=(y-\tau) /(t-\tau)$ in (4.8), we find that

$$
\inf _{y \in(\tau, t)} \operatorname{osc}_{y^{(}(y)}^{2} f \leq \iint_{D^{\prime \prime}} \mathscr{E}\left(\nabla \theta_{0}\right) d x d y \iint_{D^{\prime \prime}} \mathscr{E}(\nabla f) d x d y .
$$

Now, we note that

$$
\nabla \theta_{0}=\left(0, \frac{1}{t-\tau}\right)
$$

and therefore

$$
\iint_{D^{\prime \prime}} \mathscr{E}\left(\nabla \theta_{0}\right) d x d y=\frac{1}{(t-\tau)^{2}} \iint_{D^{\prime \prime}} \frac{A}{B} d x d y
$$

Consequently,

$$
\inf _{y \in(\tau, t)} \operatorname{osc}_{\gamma(y)}^{2} f \leq \frac{1}{(t-\tau)^{2}} \iint_{D^{\prime \prime}} \frac{A}{B} d x d y \iint_{D^{\prime \prime}} \mathscr{E}(\nabla f) d x d y .
$$

Analogously,

$$
\inf _{y \in(-t,-\tau)} \operatorname{osc}_{\gamma(y)}^{2} f \leq \frac{1}{(t-\tau)^{2}} \iint_{D^{\prime}} \frac{A}{B} d x d y \iint_{D^{\prime}} \mathscr{E}(\nabla f) d x d y .
$$

Finally,

$$
\begin{aligned}
& \inf _{y \in(\tau, t)} \operatorname{osc}_{\gamma^{(}(y)}^{2} f+\inf _{y \in(-t,-\tau)} \operatorname{osc}_{\gamma(y)}^{2} f \\
& \quad \leq \frac{1}{(t-\tau)^{2}} \max \left\{\iint_{D^{\prime}} \frac{A}{B} d x d y, \iint_{D^{\prime \prime}} \frac{A}{B} d x d y\right\} \iint_{D^{\prime} \cup D^{\prime \prime}} \mathscr{E}(\nabla f) d x d y,
\end{aligned}
$$

which proves the lemma. 
5. Energy estimates. First, we need to prove a few auxiliary statements. We let

$$
E(t)=\iint_{D(t)}\left(\frac{B}{A} f_{x}^{\prime 2}+\frac{A}{B} f^{\prime 2}\right) d x d y
$$

LEMMA 5.1. Let $f$ be a generalized solution of (2.6) with (2.8), (2.9), or (2.7) in $D(\beta)$. Then, for any $t \in(0, \beta)$,

$$
E(t) \leq \frac{1}{\int_{-\beta}^{-t} B(y) d y / \int_{y(y)} f^{2}(x, y) A(x) d x}+\frac{1}{\int_{t}^{\beta} B(y) d y / \int_{y(y)} f^{2}(x, y) A(x) d x} .
$$

Proof. Fix a Lipschitz function

$$
\psi(y)= \begin{cases}0, & \text { if } \beta<y, \\ h_{+}(y), & \text { if } t \leq y \leq \beta, \\ 1, & \text { if }-t<y<t, \\ h_{-}(y), & \text { if }-\beta \leq y \leq-t, \\ 0, & \text { if } y<-\beta\end{cases}
$$

Here, $h_{-}$and $h_{+}$are arbitrary functions for which $\psi \in \operatorname{Lip}(\mathbb{R})$.

Since $f \psi^{2}=0$ everywhere on $\gamma(-\beta) \cup \gamma(\beta)$, by (4.2) with $\phi=f \psi^{2}$ and $\Delta=D(\beta)$, we have

$$
\begin{aligned}
\iint_{D(\beta)} & \left(\left(f \psi^{2}\right)_{x}^{\prime} \frac{B}{A} f_{x}^{\prime}+\left(f \psi^{2}\right)_{y}^{\prime} \frac{A}{B} f_{y}^{\prime}\right) d x d y \\
= & \int_{\gamma(-\beta) \cup \gamma(\beta)} f \psi^{2}\left(-\frac{A}{B} f_{y}^{\prime} d x+\frac{B}{A} f_{x}^{\prime} d y\right) \\
= & 0 .
\end{aligned}
$$

From here, we find

$$
\iint_{D(\beta)} \psi^{2}\left(\frac{B}{A} f_{x}^{\prime 2}+\frac{A}{B} f_{y}^{\prime 2}\right) d x d y=-2 \iint_{D(\beta) \backslash D(t)} f \psi\left(\psi_{x}^{\prime} \frac{B}{A} f_{x}^{\prime}+\psi_{y}^{\prime} \frac{A}{B} f_{y}^{\prime}\right) d x d y
$$

Because

$$
2 f \psi\left(\psi_{x}^{\prime} \frac{B}{A} f_{x}^{\prime}+\psi_{y}^{\prime} \frac{A}{B} f_{y}^{\prime}\right) \leq 2|f \psi|^{\mathscr{E}}{ }^{1 / 2}(\nabla \psi)^{\mathscr{E}}{ }^{1 / 2}(\nabla f) \leq f^{2 \mathscr{E}}(\nabla \psi)+\psi^{2 \mathscr{E}}(\nabla f),
$$

we obtain

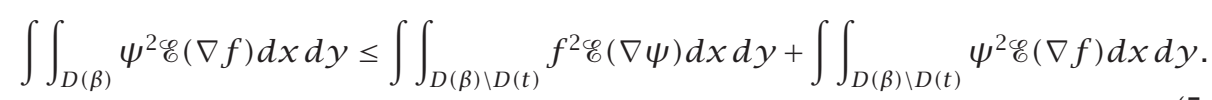


It is easy to see that $\psi_{x}^{\prime}=0$ on $D(\beta)$ and $\psi_{y}^{\prime}=h_{-}^{\prime}(y)$ for $-\beta<y<t, \psi_{y}^{\prime}=h_{+}^{\prime}(y)$ for $t<y<\beta$. So, we have

$$
\begin{aligned}
\iint_{D(t)} \mathscr{E}(\nabla f) d x d y \leq & \iint_{D \cap\{t<y<\beta\}} f^{2} h_{+}^{\prime 2} \frac{A}{B} d x d y \\
& +\iint_{D \cap\{-\beta<y<-t\}} f^{2} h_{-}^{\prime 2} \frac{A}{B} d x d y \equiv I_{1}\left(h_{+}\right)+I_{2}\left(h_{-}\right) .
\end{aligned}
$$

In order to prove the lemma, we seek the infimum of the integral $I_{1}\left(h_{+}\right)$over all functions $h_{+}, h_{+}(y)=1$ for $y \leq t$ and $h_{+}(y)=0$ for $y \geq \beta$. By the coarea formula [3, Theorem 3.2.12],

$$
I_{1}\left(h_{+}\right)=\int_{t}^{\beta} h_{+}^{\prime 2}(y) B^{-1}(y) d y \int_{\gamma(y)} f^{2}(x, y) A(x) d x .
$$

Denote

$$
\Phi(y)=B^{-1} \int_{y(y)} f^{2}(x, y) A(x) d x .
$$

But, by the Cauchy inequality,

$$
1 \leq\left(\int_{t}^{\beta} h_{+}^{\prime} d y\right)^{2} \leq \int_{t}^{\beta} h_{+}^{\prime 2} \Phi d y \int_{t}^{\beta} \frac{d y}{\Phi} .
$$

Now, we find

$$
\frac{1}{\int_{t}^{\beta} \Phi^{-1} d y} \leq \int_{t<y<\beta} h_{+}^{\prime 2} \Phi d y=I_{1}\left(h_{+}\right) .
$$

This inequality holds for any $h_{+}$, and therefore,

$$
\frac{1}{\int_{t}^{\beta} \Phi^{-1} d y} \leq \inf _{h_{+}} \int_{t<y<\beta} h_{+}^{\prime 2} \Phi d y .
$$

Choosing

$$
h_{+}(y)=\frac{\int_{y}^{\beta} \Phi^{-1}(\tau) d \tau}{\int_{t}^{\beta} \Phi^{-1}(\tau) d \tau} \text { for } t \leq y \leq \beta,
$$

we find

$$
\inf _{h_{+}} I_{1}\left(h_{+}\right)=\frac{1}{\int_{t}^{\beta} \Phi^{-1} d y} .
$$

Analogously,

$$
\inf _{h_{-}} I_{2}\left(h_{-}\right)=\frac{1}{\int_{-\beta}^{-t} \Phi^{-1} d y}
$$

Combining (5.8), (5.15), and (5.16), we arrive at (5.2). 
Next, we prove a result which may be regarded as a variant of the well-known Saint Venant's principle about a decreasing speed of energy $E(t)$ of a deformed solid (see, e.g., $[9,10,12])$.

LEMMA 5.2. If $f$ is a generalized solution of (2.6) in $D(\beta)$, then for an arbitrary $t$, $0<t<\beta$,

$$
E(t) \leq E(\beta) \exp \left\{-C \pi \int_{t}^{\beta} \kappa(t) d t\right\}
$$

where $C=1$ with boundary condition (2.9) and $C=2$ with boundary condition (2.7).

Proof. Fix $t, 0<t<\beta$. By (4.2), we can write

$$
\int_{\partial^{\prime} D(t)} f\left(-\frac{A}{B} f_{y}^{\prime} d x+\frac{B}{A} f_{x}^{\prime} d y\right)=\iint_{D(t)}\left(\frac{B}{A}\left|f_{x}^{\prime}\right|^{2}+\frac{A}{B}\left|f_{y}^{\prime}\right|^{2}\right) d x d y .
$$

From (2.9), we have

$$
-\int_{\gamma(-t) \cup y(t)} f \frac{A}{B} f_{y}^{\prime} d x=\iint_{D(t)}\left(\frac{B}{A}\left|f_{x}^{\prime}\right|^{2}+\frac{A}{B}\left|f_{y}^{\prime}\right|^{2}\right) d x d y,
$$

that is,

$$
E(t) \leq B^{-1}(-t) \int_{\gamma(-t)}|f|\left|A f_{y}^{\prime}\right| d x+B^{-1}(t) \int_{\gamma(t)}|f|\left|A f_{y}^{\prime}\right| d x .
$$

We estimate the first term in the right side of this inequality. By the Hölder inequality,

$$
\int_{\gamma(-t)}|f|\left|A f_{y}^{\prime}\right| d x \leq\left(\int_{\gamma(-t)} A|f|^{2} d x\right)^{1 / 2}\left(\int_{\gamma(-t)} A\left|f_{y}^{\prime}\right|^{2} d x\right)^{1 / 2} .
$$

For an arbitrary $t \in(-\beta, \beta)$, we set

$$
\lambda_{1}(t)=\inf _{\phi}\left(\frac{\int_{y(t)} A^{-1}(x)\left|\phi^{\prime}(x)\right|^{2} d x}{\int_{\gamma(t)} A(x) \phi^{2}(x) d x}\right)^{1 / 2}
$$

where the infimum is taken over all Lipschitz on $\gamma(t)$ functions $\phi, \phi=0$, at $\Gamma_{0} \cap \overline{\gamma(t)}$.

The function $f(x,-t)$ vanishes at $\Gamma_{0} \cap \gamma(-t)$. From (5.22), it follows that

$$
\int_{\gamma(-t)} A(x)|f(x,-t)|^{2} d x \leq \frac{1}{\lambda_{1}^{2}(-t)} \int_{\gamma(-t)} A^{-1}(x)\left|f_{x}^{\prime}\right|^{2} d x .
$$

Now, we obtain

$$
\int_{\gamma(-t)}|f(x,-t)| \frac{A}{B}\left|f_{y}^{\prime}\right| d x \leq \frac{B^{-1}(t)}{\lambda_{1}(-t)}\left(\int_{\gamma(-t)} \frac{B}{A}\left|f_{x}^{\prime}\right|^{2} d x\right)^{1 / 2}\left(\int_{\gamma(-t)} \frac{A}{B}\left|f_{x}^{\prime}\right|^{2} d x\right)^{1 / 2} .
$$

By the inequality

$$
2 a b \leq a^{2}+b^{2}
$$


we find

$$
\int_{y(-t)}|f| \frac{A}{B}\left|f_{y}^{\prime}\right| d x \leq \frac{B^{-1}(-t)}{2 \lambda_{1}(-t)} \int_{y(-t)}\left(\frac{B}{A}\left|f_{x}^{\prime}\right|^{2}+\frac{A}{B}\left|f_{y}^{\prime}\right|^{2}\right) d x .
$$

Analogously,

$$
\int_{\gamma^{(}(t)}|f| \frac{A}{B}\left|f_{y}^{\prime}\right| d x \leq \frac{B^{-1}(t)}{2 \lambda_{1}(t)} \int_{\gamma^{(}(t)}\left(\frac{B}{A}\left|f_{x}^{\prime}\right|^{2}+\frac{A}{B}\left|f_{y}^{\prime}\right|^{2}\right) d x
$$

Adding both inequalities, we get

$$
\int_{\eta(t)}|f| \frac{A}{B}\left|f_{y}^{\prime}\right| d x \leq \max \left\{\frac{B^{-1}(-t)}{2 \lambda_{1}(-t)}, \frac{B^{-1}(t)}{2 \lambda_{1}(t)}\right\} \int_{\eta(t)}\left(\frac{B}{A}\left|f_{x}^{\prime}\right|^{2}+\frac{A}{B}\left|f_{y}^{\prime}\right|^{2}\right) d x,
$$

where

$$
\eta(t)=\gamma(-t) \cup \gamma(t)
$$

The quantity $\lambda_{1}(t)$ in (5.22) is easily computable. Let $x_{0} \in \overline{\gamma(t)}$ be the left end of $\gamma(t)$ and let

$$
u=\int_{x_{0}}^{x} A(t) d t
$$

We have

$$
\int_{\gamma^{(t)}} A(x) \phi^{2}(x) d x=\int_{0}^{L(t)} \tilde{\phi}^{2}(u) d u
$$

where

$$
\tilde{\phi}(u)=\phi\left(x^{-1}(u)\right), \quad L(t)=\int_{\gamma(t)} A(x) d x
$$

Because

$$
\tilde{\phi}^{\prime}(u)=\phi^{\prime}\left(x^{-1}(u)\right) \frac{1}{A(x(u))},
$$

we find

$$
\int_{\gamma(t)} \frac{1}{A(x)}\left|\phi^{\prime}(x)\right|^{2} d x=\int_{0}^{L(t)} \tilde{\phi}^{\prime 2}(u) d u .
$$

Thus, from (5.22), we obtain

$$
\lambda_{1}(t)=\inf _{\phi}\left(\frac{\int_{0}^{L(t)}\left|\phi^{\prime}(u)\right|^{2} d u}{\int_{0}^{L(t)} \phi^{2}(u) d u}\right)^{1 / 2},
$$

where the infimum is taken over all Lipschitz functions $\phi$ defined on $(0, L(t))$ with $\phi(0)=0$. 
If $f$ satisfies (2.6) with (2.7), these arguments remain unchanged if we replace $\lambda_{1}(t)$ by

$$
\lambda_{2}(t)=\inf _{\psi}\left(\frac{\int_{\gamma(t)} A^{-1}(x)\left|\psi^{\prime}(x)\right|^{2} d x}{\int_{\gamma^{(}(t)} A(x) \psi^{2}(x) d x}\right)^{1 / 2},
$$

where the infimum is taken over all functions $\psi$ that are Lipschitz on $\gamma(t)$ such that $\psi$ equals to 0 on both ends of $\gamma(t)$.

As (5.35) may be rephrased as

$$
\lambda_{2}(t)=\inf _{\psi}\left(\frac{\int_{0}^{L(t)}\left|\psi^{\prime}(u)\right|^{2} d u}{\int_{0}^{L(t)} \psi^{2}(u) d u}\right)^{1 / 2},
$$

where the infimum is taken over all functions $\psi$ Lipschitz on $(0, L(t))$ and $\psi(0)=$ $\psi(L(t))=0$. So, by the Wirtinger inequality (e.g., see, [1, Theorem 7, Chapter V]), we have the well-known relation

$$
\lambda_{2}(t)=\frac{\pi}{L(t)}
$$

Analogously, for calculation of $\lambda_{1}(t)$ at first, we extend $\phi(x)$ on $(0,2 L(t))$ by symmetry such that

$$
\phi^{*}(x)=\phi^{*}(2 L-x),\left.\quad \phi^{*}(x)\right|_{(0, L(t))}=\phi(x) .
$$

Now, we have $\phi^{*}(0)=\phi^{*}(2 L(t))=0$ and as (5.38), we establish

$$
\lambda_{1}(t)=\frac{\pi}{2 L(t)}
$$

We note

$$
E^{\prime}(t)=\int_{\gamma(-t) \cup \gamma(t)}\left(\frac{B}{A}\left|f_{x}^{\prime}\right|^{2}+\frac{A}{B}\left|f_{y}^{\prime}\right|^{2}\right) d x
$$

In the case (2.9), using (5.20), (5.28), and (5.40), we arrive at

$$
E(t) \leq \frac{1}{\pi \kappa(t)} E^{\prime}(t)
$$

Integrating (5.42), we have the inequality

$$
\log \frac{E(\beta)}{E(t)} \geq 2 \int_{t}^{\beta} \pi \kappa(t) d t
$$

which implies (5.17) with $C=1$.

In the case (2.7) from (5.35) and (5.38) as above, we prove (5.17) with $C=2$.

6. Proof of main theorems. First, we consider the proof of Theorem 3.1. We note that the proof of Theorem 3.2 follows easily from Theorem 3.1 and Lemma 5.2. 
Let $m=\min _{\gamma(-\beta) \cup \gamma(\beta)} f$ and $M=\max _{\gamma(-\beta) \cup \gamma(\beta)} f$. Consider a new function $f^{*}=f-$ $(m+M) / 2$. It is easy to see that the function $f^{*}$ satisfies (2.9) and

$$
\max _{\gamma(-\beta) \cup \gamma_{(\beta)}}\left|f^{*}(x, y)\right| \leq \frac{1}{2} \operatorname{osc}_{\gamma(-\beta) \cup \gamma(\beta)} f(x, y)=\frac{\Omega}{2} .
$$

Without loss of generality, we may assume that the solution $f$ also satisfies the above inequality and, consequently, by Lemma 4.1,

$$
\max _{D(\beta)}|f(x, y)| \leq \frac{\Omega}{2} \text {. }
$$

Fix arbitrarily $\epsilon>0$. Choose $\tau=t-1$ in (4.20) and let $y_{1} \in(-t,-t+1), y_{2} \in(t-1, t)$ such that

$$
\operatorname{osc}_{\gamma\left(y_{1}\right)}^{2} f \leq \inf _{y \in(-t,-t+1)} \operatorname{osc}_{\gamma(y)}^{2} f+\frac{\epsilon}{3}, \quad \operatorname{osc}_{\gamma\left(y_{2}\right)}^{2} f \leq \inf _{y \in(t-1, t)} \operatorname{osc}_{\gamma(y)}^{2} f+\frac{\epsilon}{3},
$$

and $s_{0} \in(0,1)$ such that

$$
\operatorname{osc}_{\Gamma_{S_{0}}(t)}^{2} f \leq \inf _{0 \leq s \leq 1} \operatorname{osc}_{\Gamma_{s}(t)}^{2} f+\frac{\epsilon}{3}
$$

Using Lemma 4.1, we have

$$
\begin{aligned}
\operatorname{osc}_{D(t-1)} f & \leq \operatorname{osc}_{\partial D(t-1)} f \leq \operatorname{osc}_{\gamma(-t+1) \cup \gamma(t-1)} f \\
& \leq \operatorname{osc}_{\gamma\left(y_{1}\right)} f+\operatorname{osc}_{\gamma\left(y_{2}\right)} f+\operatorname{osc}_{\Gamma_{S_{0}}(t)} f,
\end{aligned}
$$

or

$$
\operatorname{osc}_{D(t-1)}^{2} f \leq 3\left(\operatorname{osc}_{\gamma\left(y_{1}\right)}^{2} f+\operatorname{osc}_{\gamma\left(y_{2}\right)}^{2} f+\operatorname{osc}_{\Gamma_{S_{0}}(t)}^{2} f\right) .
$$

Hence,

$$
\operatorname{osc}_{D(t-1)}^{2} f \leq 3\left(\inf _{y \in(-t,-t+1)} \operatorname{osc}_{\gamma(y)}^{2} f+\inf _{y \in(t-1, t)} \operatorname{osc}_{\gamma(y)}^{2} f+\inf _{0 \leq s \leq 1} \operatorname{osc}_{\Gamma_{s}(t)}^{2} f+\epsilon\right) .
$$

Now, from (4.8) and (4.20), it follows that

$$
\begin{gathered}
\operatorname{osc}_{D(t-1)}^{2} f \leq 3\left(\max \left\{\iint_{D^{*}} \frac{A}{B} d x d y, \iint_{D^{* *}} \frac{A}{B} d x d y\right\} \iint_{D(t) \backslash D(t-1)} \mathscr{E}(\nabla f) d x d y\right. \\
\left.+\iint_{D(t)} \mathscr{E}(\nabla \theta) d x d y \iint_{D(t)} \mathscr{E}(\nabla f) d x d y+\epsilon\right) .
\end{gathered}
$$

Since $\epsilon>0$ is arbitrary,

$$
\operatorname{osc}_{D(t-1)}^{2} f \leq 3\left(\max \left\{\iint_{D^{*}} \frac{A}{B} d x d y, \iint_{D^{* *}} \frac{A}{B} d x d y\right\}+\iint_{D(t)} \mathscr{E}(\nabla \theta) d x d y\right) E(t),
$$

or

$$
\operatorname{osc}_{D(t-1)}^{2} f \leq 3(e(t)+\mu(t)) E(t) .
$$


The estimate (5.2) implies

$$
E(t) \leq \frac{2}{\min \left\{\int_{t}^{\beta} B(y) d y / \int_{\gamma(y)} f^{2}(x, y) A(x) d x, \int_{-\beta}^{-t} B(y) d y / \int_{\gamma(y)} f^{2}(x, y) A(x) d x\right\}} .
$$

Consequently, by (6.2)

$$
E(t) \leq \frac{\Omega^{2}}{2 \min \left\{\int_{t}^{\beta} B(y) d y / \int_{\gamma(y)} A(x) d x, \int_{-\beta}^{-t} B(y) d y / \int_{y(y)} A(x) d x\right\}},
$$

and we find

$$
E(t) \leq \frac{\Omega^{2}}{2 I(t, \beta)}
$$

Combining (6.10) and (6.13), we arrive at the following estimate:

$$
\operatorname{osc}_{D(t-1)}^{2} f \leq \frac{3}{2}(e(t)+\mu(t)) \frac{\Omega^{2}}{I(t, \beta)} .
$$

Thus, the assumption (3.5) implies from (6.14) that

$$
\operatorname{osc}_{D(t-1)} f \leq s,
$$

that is, the subdomain $D(t-1)$ is an $s$-zone, which is the result of Theorem 3.1.

To prove Theorem 3.2, we combine the estimate (6.10) with estimates (5.17) in Lemma 5.2. We obtain, in the case of the condition (2.9),

$$
\operatorname{osc}_{D(t-2)}^{2} f \leq 3(e(t-1)+\mu(t-1)) E(\beta-1) \exp \left\{-\pi \int_{t-1}^{\beta-1} \kappa(\tau) d \tau\right\}
$$

or, if $f$ satisfies (2.7),

$$
\operatorname{osc}_{D(t-2)}^{2} f \leq 3(e(t-1)+\mu(t-1)) E(\beta-1) \exp \left\{-2 \pi \int_{t-1}^{\beta-1} \kappa(\tau) d \tau\right\} .
$$

As in (6.13), we find

$$
E(\beta-1) \leq \frac{\Omega^{2}}{2 I(\beta-1, \beta)}
$$

Estimates (6.16), (6.17), and (6.18) imply, respectively,

$$
\begin{aligned}
& \operatorname{osc}_{D(t-2)}^{2} f \leq \frac{3}{2 I(\beta-1, \beta)}(e(t-1)+\mu(t-1)) \exp \left\{-\pi \int_{t-1}^{\beta-1} \kappa(\tau) d \tau\right\}, \\
& \operatorname{osc}_{D(t-2)}^{2} f \leq \frac{3}{2 I(\beta-1, \beta)}(e(t-1)+\mu(t-1)) \exp \left\{-2 \pi \int_{t-1}^{\beta-1} \kappa(\tau) d \tau\right\} .
\end{aligned}
$$

From assumptions (3.7), we conclude that $D(t-2)$ is an $s$-zone. 
7. Examples. To illustrate our results, we will describe some examples of surfaces defined by almost Liouville line elements and then consider the application of the main theorems to a boundary value problem over conic belts.

Consider an almost Liouville line element defined as follows. Let $\mathscr{D}=\left(D, d s^{2}\right)$ be a surface obtained by rotation of the graph of a function $u=\rho(w)$ around the $w$-axis. We assume that $\rho(w)$ is defined on a segment $0 \leq w \leq \rho_{0}$, nonnegative and belongs to $C^{2}\left[0, \rho_{0}\right]$.

We set $y=\xi(w)$ to be the arc length function

$$
y=\xi(w)=\int_{0}^{w} \sqrt{1+\rho^{\prime 2}(\tau)} d \tau
$$

and let $w=\xi^{-1}(y)=\eta(y)$ be its inverse function.

We introduce special coordinates $(x, y)$ of a point $(u, v, w)$ on $\mathscr{D}$ by setting

$$
x=\tan ^{-1} \frac{v}{u}, \quad y=\xi(w)
$$

If we denote $r(y)=\rho \circ \eta(y)$, then it is easy to see that

$$
u=r(y) \cos x, \quad v=r(y) \sin x, \quad w=\eta(y) .
$$

Clearly, the lines $x=$ const and $y=$ const on $\mathscr{D}$ are orthogonal to each other. Therefore, for the length element $d s$ on $\mathscr{D}$, we have

$$
d s^{2}=r^{2}(y) d x^{2}+d y^{2}
$$

Consequently, we have

$$
Q(x, y)=r(y), \quad A(x)=1, \quad B(y)=\frac{1}{r(y)},
$$

and so $\mathscr{D}$ belongs to the class of surfaces defined by almost Liouville line elements.

We set by

$$
\left|\nabla_{\mathscr{D}} f\right|^{2}=Q^{-2}(x, y)\left(\frac{f_{x}^{\prime 2}}{A^{2}}+\frac{f_{y}^{\prime 2}}{B^{2}}\right)
$$

the square of the gradient of a function $f$ on $\mathscr{D}$, and, next,

$$
\mathscr{E}(\nabla f)=\frac{B}{A} f_{x}^{\prime 2}+\frac{A}{B} f_{y}^{\prime 2}
$$

With the aid of (2.5) and (7.6), the basic variational problem

$$
\min _{f} \iint_{D}\left|\nabla_{\mathscr{D}} f\right|^{2} d \sigma
$$


to be investigated may be written as

$$
\min _{f} \iint_{D} \mathscr{E}(\nabla f) d x d y
$$

and the corresponding Laplace-Beltrami equation on $\mathscr{D}=\left(D, d s^{2}\right)$ has the form (2.6).

Since $Q=r(y), A=1$, and $B=1 / r(y)$ for the surface of revolution considered above, the corresponding Laplace-Beltrami equation has the form

$$
\frac{1}{r(y)} \frac{\partial}{\partial x}\left(\frac{\partial f}{\partial x}\right)+\frac{\partial}{\partial y}\left(r(y) \frac{\partial f}{\partial y}\right)=0 .
$$

By choosing $\rho=1+w, 0 \leq w \leq \sqrt{2} \beta$, we have

$$
r(y)=1+k y, \quad k=\frac{1}{\sqrt{2}} .
$$

Fix $\alpha, 0<\alpha<2 \pi$. The resulting surface of revolution is a conic belt defined by the parametric equations

$$
\begin{gathered}
u=(1+k y) \cos x, \\
v=(1+k y) \sin x, \\
w=k y,
\end{gathered}
$$

where $0<x<\alpha, 0 \leq y \leq 2 \beta$.

Set the rectangle

$$
\Delta=\{0<x<\alpha, 0<y<2 \beta\}
$$

and consider the following boundary problem.

(P) Find the solution of (7.10) subject to (2.8), ((2.9) or (2.7)) on vertical parts of $\Delta$ and

$$
\left.f\right|_{y=0}=1,\left.\quad f\right|_{y=2 \beta}=2
$$

on horizontal parts.

Introducing new variables

$$
\tilde{x}=\frac{x}{\alpha}, \quad \tilde{y}=y-\beta,
$$

if $(x, y) \in \Delta$, then rescaling to $(\tilde{x}, \tilde{y})$, we have

$$
D(\beta)=\{(\tilde{x}, \tilde{y}): 0<\tilde{x}<1,-\beta<\tilde{y}<\beta\} .
$$

Here, $\theta=\tilde{x}$, so,

$$
\begin{aligned}
\Gamma_{0}(\beta) & =\{(\tilde{x}, \tilde{y}) \in \partial D(\beta): \tilde{x}=0\}, & \Gamma_{1}(\beta)=\{(\tilde{x}, \tilde{y}) \in \partial D(\beta): \tilde{x}=1\}, \\
\gamma(-\beta) & =\{(\tilde{x}, \tilde{y}) \in \partial D(\beta): \tilde{y}=-\beta\}, & \gamma(\beta)=\{(\tilde{x}, \tilde{y}) \in \partial D(\beta): \tilde{y}=\beta\} .
\end{aligned}
$$


From (3.1) and (7.14), we have $\Omega=1$. Consequently, for the boundary value problem (7.10)-(7.14), we have from Theorem 3.1, (3.2), (3.3), (3.4),

$$
\begin{aligned}
e(t) & =\frac{1}{\alpha k} \log \frac{1+k \beta+k t}{1+k \beta-k t} ; \\
\mu(t) & =\alpha\left(1+\frac{k}{2}+k \beta+k t\right) ; \\
I(t, \beta) & =\frac{1}{\alpha k} \log \frac{1+2 k \beta}{1+k \beta+k t} ;
\end{aligned}
$$

and thus, according to Theorem 3.1, for any given $s>0$, if $t \in(1, \beta)$ is such that

$$
\frac{1}{\alpha k} \log \frac{1+k \beta+k t}{1+k \beta-k t}+\alpha\left(1+\frac{k}{2}+k \beta+k t\right) \leq \frac{3}{2} \frac{s^{2}}{\alpha k} \log \frac{1+2 k \beta}{1+k \beta+k t}
$$

then the band $D(t-1)$ is an $s$-zone.

Similarly, from (3.2), (3.3), (3.4), (3.6),

$$
\begin{aligned}
e(t-1) & =\frac{1}{\alpha k} \log \frac{1+k \beta+k(t-1)}{1+k \beta-k(t-1)} ; \\
\mu(t-1) & =\alpha\left(1+\frac{k}{2}+k \beta+k(t-1)\right) ; \\
I(\beta-1, \beta) & =\frac{1}{\alpha k} \log \frac{1+2 k \beta}{1+k \beta+k(\beta-1)} ; \\
L(t) & =\alpha, \quad \kappa(t)=\frac{1}{1+k \beta+k t} ; \\
\int_{t-1}^{\beta-1} \kappa(\tau) d \tau & =\int_{t-1}^{\beta-1} \frac{1}{1+k \beta+k T} d \tau=\frac{1}{k} \log \frac{1+k \beta+k(\beta-1)}{1+k \beta+k(t-1)} ;
\end{aligned}
$$

we see from Theorem 3.2 that for any given $s>0$, if $t \in(2, \beta)$ is such that

$$
\begin{aligned}
& \left(\frac{1}{\alpha k} \log \frac{1+k \beta+k(t-1)}{1+k \beta-k(t-1)}+\alpha\left(1+\frac{k}{2}+k \beta+k(t-1)\right)\right) \\
& \quad \cdot \exp \left(-C \pi \frac{1}{k} \log \frac{1+k \beta+k(\beta-1)}{1+k \beta+k(t-1)}\right) \leq \frac{2}{3} \frac{s^{2}}{\alpha k} \log \frac{1+2 k \beta}{1+k \beta+k(\beta-1)},
\end{aligned}
$$

then $D(t-2)$ is an $s$-zone.

ACKNOWLEDGMENTS. The work of the first author is partially supported by INTAS, Project 10170. Main results of this article were obtained when the authors were working at the Department of Mathematics of Brigham Young University. The first author wishes to thank leaders of BYU for hospitality. We also thank Dr. Steven McKay for his comments. We also thank the referee for their many suggestions and for pointing out a simpler proof of (4.12) in Lemma 4.2, which we have reproduced in this paper. 


\section{REFERENCES}

[1] E. F. Beckenbach and R. Bellman, Inequalities, Ergebnisse der Mathematik und ihrer Grenzgebiete, Springer-Verlag, Berlin, 1961.

[2] F. Braudel, Le Temps du Monde, Civilisation Matérielle, Économie et Capitalisme, XV-XVIII siécle, vol. 3, Armand Colin, Paris, 1979.

[3] H. Federer, Geometric Measure Theory, Die Grundlehren der mathematischen Wissenschaften, vol. 153, Springer-Verlag, New York, 1969.

[4] D. Gaier, Ermittlung des konformen Moduls von Vierecken mit Differenzenmethoden, Numer. Math. 19 (1972), 179-194 (German).

[5] W. Klingenberg, A Course in Differential Geometry, Graduate Texts in Mathematics, Springer-Verlag, New York, 1978.

[6] R. Menikoff and C. Zemach, Methods for numerical conformal mapping, J. Comput. Phys. 36 (1980), no. 3, 366-410.

[7] V. M. Miklyukov, Stagnation zones of solutions to the Laplace-Beltrami equation in long strips, Siberian Adv. Math. 12 (2002), no. 3, 62-78.

[8] _ s-Zones of harmonic functions on narrow and long bands, Mathematical and Application Analysis, Tyumen State University, Tyumen, 2003, pp. 89-118.

[9] V. M. Miklyukov and V. G. Tkachev, Denjoy-Ahlfors theorem for harmonic functions on Riemannian manifolds and external structure of minimal surfaces, Comm. Anal. Geom. 4 (1996), no. 4, 547-587.

[10] O. A. Oleĭnik, Some mathematical problems of elasticity theory, Current Problems in Mathematical Physics and Numerical Mathematics, "Nauka”, Moscow, 1984, pp. 144-160, 214.

[11] L. N. Trefethen, Numerical computation of the Schwarz-Christoffel transformation, SIAM J. Sci. Statist. Comput. 1 (1980), no. 1, 82-102.

[12] S. Vidoli, R. C. Batra, and F. dell'Isola, Saint-Venant's problem for a second-order piezoelectric prismatic bar, Internat. J. Engrg. Sci. 38 (2000), no. 1, 21-45.

V. M. Miklyukov: Mathematics Department, Volgograd State University, 400062 Volgograd, Russia

E-mail address: mik1yuk@v1ink.ru

S.-S. Chow: Mathematics Department, Brigham Young University, Provo, UT 84602, USA

E-mail address: schow@math. byu. edu

V. P. Solovjov: Mathematics Department, Brigham Young University, Provo, UT 84602, USA

E-mail address: vps@et.byu.edu 


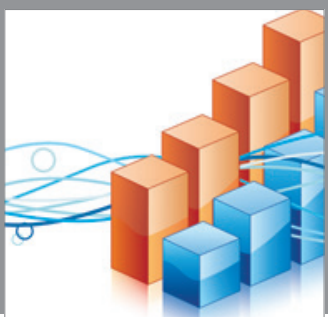

Advances in

Operations Research

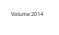

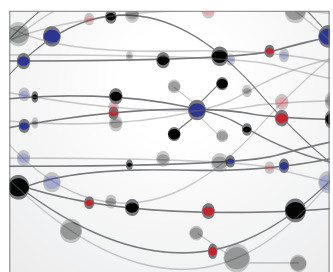

\section{The Scientific} World Journal
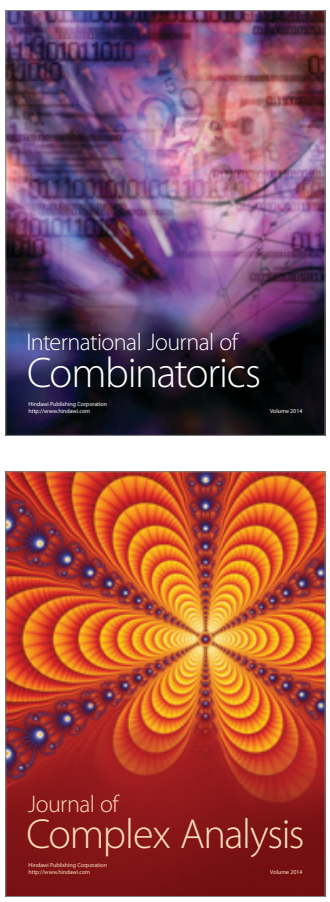

International Journal of

Mathematics and

Mathematical

Sciences
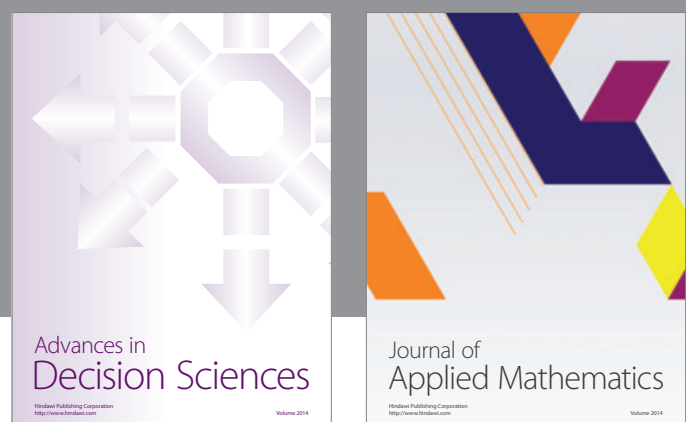

Journal of

Applied Mathematics
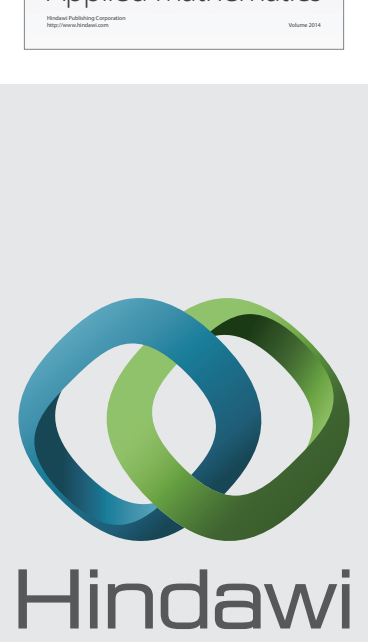

Submit your manuscripts at http://www.hindawi.com
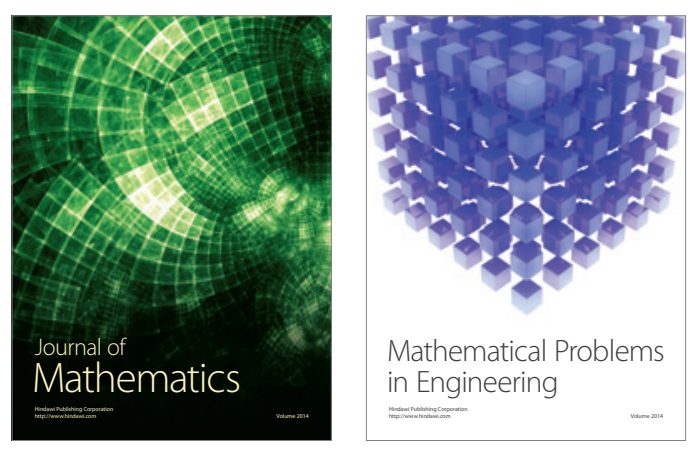

Mathematical Problems in Engineering
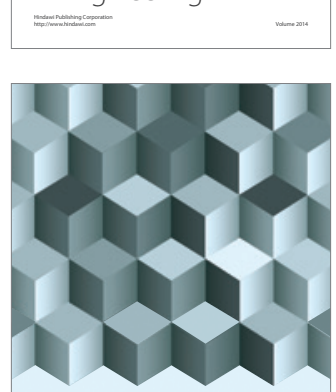

Journal of

Function Spaces
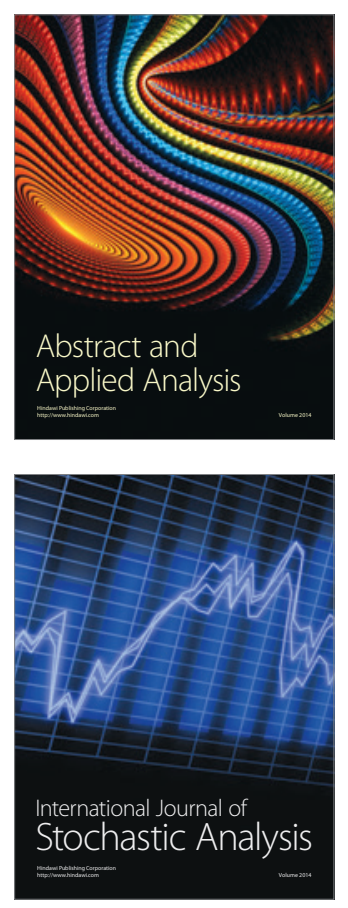

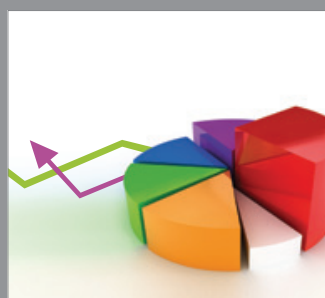

ournal of

Probability and Statistics

Promensencen
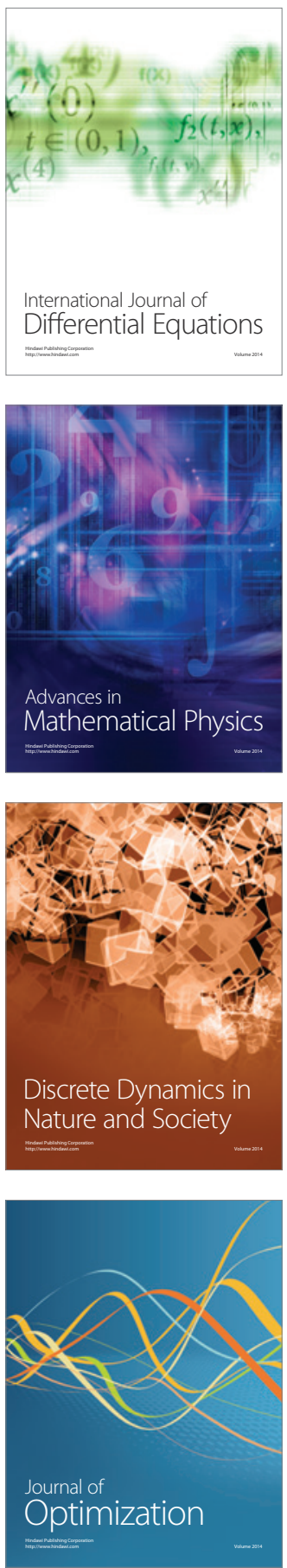